\title{
Bilinear design of fully differential switched-capacitor ladder filters
}

\author{
Prof. F. Maloberti, Mem. I.E.E.E., A.E.I., Prof. F. Montecchi, Mem. I.E.E.E., \\ A.E.I., G. Torelli, Dr. Eng., and E. Halász, Ph.D., Cand.Eng.Sci.
}

Indexing terms: Filters and filtering, Circuit theory and design

\begin{abstract}
Fully differential switched-capacitor (SC) analogue blocks are shown to be suitable for implementing $\mathrm{SC}$ ladder filters via the bilinear transform of the corresponding analogue $R L C$ passive prototypes. These filters become fully insensitive to parasitic capacitance effects, while the interesting features of the differential implementation, i.e. power supply and common mode rejection ratios and dynamic range improvements, are retained. The technique presented is useful for the exact design of wide-band SC filters, i.e. for filters with a small ratio between the clock frequency and the cut-off frequency. As an example, the bilinear design of an 8th-order Cauer bandpass filter is given, with numerically computed results.
\end{abstract}

1

\section{Introduction}

The most widely used technique for designing high-order switched-capacitor (SC) filters has been the one which refers to the flow-graph description of an analogue $R L C$ passive prototype, generally considered as a doubly terminated $L C$ lossless ladder chain [1]. This method follows the technique used in the past for the design of active ladder filters (active leapfrog structures) [2].

The active ladder configuration is currently regarded as the optimum choice for high-selectivity SC filter design, as the very low sensitivity of the passband response with respect to the component variations [3] is retained in SC implementations as well as in the original passive prototype and in its continuous-time active realisations [4].

Presently, SC implementations are derived from the flow-graph description by using the concept of the 'lossless discrete integrator' (LDI) [5], or, in other words, by using the LDI transformation, which relates the complex frequency variable $s$ of the analogue prototype to the complex variable $z$ of the sampled-data system, according to the following relationship:

$$
s \rightarrow \frac{1}{T}\left(z^{1 / 2}-z^{-1 / 2}\right)
$$

where $T$ represents the sampling period.

The LDI transformation works quite well so long as the sampling frequency $f_{s} \equiv 1 / T$ is much higher than the cut-off frequency $f_{h}$. A ratio $f_{s} / f_{h}$ in the range of 30 to 50 has generally been considered in classical implementations for the voice frequency range (PCM filters) [6].

Such a large ratio $f_{s} / f_{h}$ is required as the LDI transformation cannot be physically implemented in the terminating sections of the ladder chain, where damped integrators are needed to simulate the load and source resistors of the analogue prototype [4]. This aspect is generally referred as the 'termination error', because of the extra phase shift in the damping loop of the actual damped SC integrators with respect to the required one [7].

To overcome this shortcoming, predistortion methods have been used [8]. Alternative design techniques can be

Paper 4207G (E10), first received 15th January and in revised form 1st August 1985 Professors Maloberti and Montecchi are with the Dipartimento di Elettronica, Universita di Pavia, Corso Strada Nuova 106/c, 27100 Pavia, Italy. Dr. Torelli is with SGS-Microelettronica, Agrate Brianza (MI), Italy. Dr. Halász is with the Institute of Telecommunication \& Electronics, Technical University of Budapest, Stoczek u.2, Budapest H-1111, Hungary followed to obtain the exact implementations [9-12]. In general, these techniques are quite cumbersome to use, and the simplest satisfactory technique is the one that uses both LDI and 'bilinear' transforms [13]. More recently, exact design methods of LDI structures have been given, referring to standard tables for wave digital filters [14].

In contrast to the LDI transformation, the bilinear $s$ to $z$ transformation,

$$
s \rightarrow \frac{2}{T} \frac{z-1}{z+1}
$$

can avoid any termination error when used for SC ladder filters, as its application can also be extended for damped integrators. Frequency warping effects will be introduced, but their presence can easily be taken into account by means of prewarping techniques [4].

Unfortunately, first-order SC blocks bilinearly referred to their continuous-time active counterpart are parasitic sensitive [15] or, when not, they require equally valued capacitors or multiphase clocking schemes $[16,17]$.

A 'bilinear SC resistor' could represent an optimum solution which combines the advantageous properties of both the leapfrog circuit and the $s$ to $z$ bilinear mapping. Moreover, this is achieved by means of an immediate and easy design of all the time constants of the flow-graph integrators [18].

In the present work, we show how the fully differential structure can be used to obtain positive and/or negative $\mathrm{SC}$ resistors, so as to have available positive and/or negative parastitic insensitive bilinear integrators (both damped and not damped), to be used as basic building blocks for an SC leapfrog design.

The differential approach presented here is simpler than the alternative bilinear design technique of SC ladder filters given in Reference 19.

In Section 2, the basic bilinear SC circuits are presented, in Section 3 the design procedure is given, and in Section 4 the design of an 8th-order Cauer bandpass filter is shown as an example.

\section{Bilinear differential integrators}

The basic building blocks useful for implementing SC ladder filters via flow-graph techniques are the first-order integrators, positive and negative, with or without a 'resistive' damping. If the design implies transmission zeros, the summing operation is also required, together with the integration of other input signals. 
In Fig. 1A, the classical continuous-time active integrator is shown. Its transfer function is given by:

$$
H_{a 1}(s)=-\frac{1}{s R_{1} C_{2}}
$$

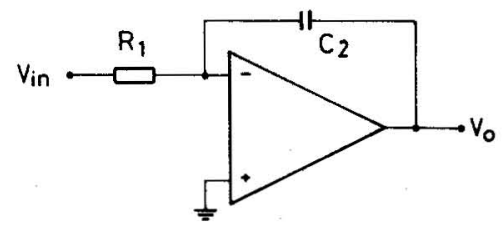

Fig. 1A Continuous-time active integrator

If a positive integrator is required, an inverter has to be added. This is no longer necessary if a fully differential implementation is used, as shown in Fig. 1B, where the

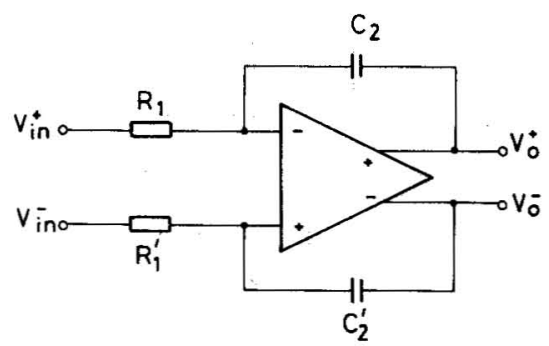

Fig. 1B Differential active integrator

operational amplifier can be, for example, of the type considered in Reference 25 . Here $\left(V_{\text {in }}^{+}, V_{\text {in }}^{-}\right)$represent the differential input signal pair, and $\left(V_{o}^{+}, V_{o}^{-}\right)$the differential output signal pair. If the input signal has no common mode terms, i.e. $V_{i n}^{+}=-V_{i n}^{-}$, the transfer function of the structure of Fig. 1B is given by

$$
\begin{aligned}
H_{a 1}^{\prime}(s) \equiv & \frac{V_{o}^{+}(s)-V_{o}^{-}(s)}{V_{i n}^{+}(s)-V_{i n}^{-}(s)} \\
& =-\frac{2+s\left(\tau+\tau^{\prime}\right)}{2 s^{2} \tau \tau^{\prime}+s\left(\tau+\tau^{\prime}\right)}
\end{aligned}
$$

with $\tau=R_{1} C_{2}$ and $\tau^{\prime}=R_{1}^{\prime} C_{2}^{\prime}$. If now $\tau^{\prime} \equiv \tau$, eqn. $4 a$ becomes

$$
H_{\mathrm{a} 1}^{\prime}(s)=-\frac{1}{s R_{1} C_{2}}
$$

which is identical to eqn. 3 .

To obtain a positive integrator, the connection of the input signal pair is reversed, whereas the output is taken out with the same polarity as above. This is a natural consequence of the differential topology, where voltages of both polarity are available at each stage. Depending on the connection between different stages, positive or negative transfer functions can be obtained.

In Fig. 1C, the bilinear SC integrator is shown in the

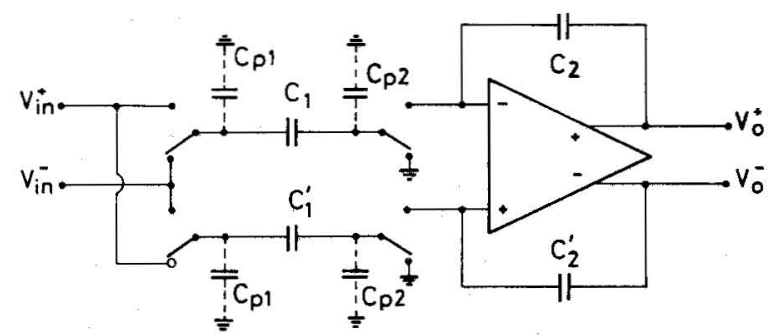

Fig. 1C Bilinear SC active integrator fully differential form by using a differential operational amplifier of the type reported, for example, in Reference 21; a common mode feedback internal to the operational amplifier ensures that at any instant $V_{o}^{+}=-V_{o}^{-}$.

The bilinear SC integrator of Fig. 1C uses parasitic insensitive SC structures, being each node of each capacitor switched between two voltage sources, or between ground and virtual ground [20]. Assuming that the input voltages do not change for an overall sampling period $T$, we can write

$$
\begin{aligned}
V_{o}^{+}[(n+1) T]= & V_{o}^{+}(n T)-\alpha\left\{V_{i n}^{+}[(n+1) T]-V_{i n}^{-}(n T)\right\} \\
& +(1+\alpha) V_{x}[(n+1) T]-V_{x}(n T) \quad(5 a) \\
V_{o}^{-}[(n+1) T]= & V_{o}^{-}(n T)-\alpha^{\prime}\left\{V_{i n}^{-}[(n+1) T]-V_{i n}^{+}(n T)\right\} \\
& +\left(1+\alpha^{\prime}\right) V_{x}[(n+1) T]-V_{x}(n T) \quad(5 b)
\end{aligned}
$$

with $\alpha=C_{1} / C_{2}, \alpha^{\prime}=C_{1}^{\prime} / C_{2}^{\prime}$ and $V_{x}$ being the voltage of the virtual ground nodes.

Taking the $z$-transform of eqns. $5 a$ and $5 b$, under the assumption that the input signal has no common mode term, we obtain

$$
\begin{aligned}
H_{1}(z) & \equiv \frac{V_{o}^{+}(z)-V_{o}^{-}(z)}{V_{i n}^{+}(z)-V_{i n}^{-}(z)} \\
& =-\frac{(z+1)}{(z-1)} \frac{z\left(2 \alpha \alpha^{\prime}+\alpha+\alpha^{\prime}\right)-\alpha-\alpha^{\prime}}{z\left(2+\alpha+\alpha^{\prime}\right)-2}
\end{aligned}
$$

If the positive and negative paths are well matched, i.e. $\alpha=\alpha^{\prime}$, we obtain

$$
H_{1}(z)=-\frac{C_{1}}{C_{2}}\left(\frac{z-1}{z+1}\right)
$$

A positive transfer function is obtained by reversing the input connection as in the differential continuous-time case.

Eqn. $6 b$ can be recognised as being the transfer function of a sampled-data bilinear integrator, which in turn can be regarded as having been derived from eqn. 3 or eqn. $4 b$, by substituting $s$ with the bilinear transform given in eqn. 2 , using

$$
C_{1}=\frac{T}{2 R_{1}}
$$

and maintaining the integrating capacitor $C_{2}$ at the same value as in the continuous time case. Such a bilinear SC integrator has been independently considered by other authors [21].

In Fig. 2A, an active continuous-time damped integrator is shown. Its transfer function is given by

$$
H_{a 2}(s)=-\frac{R_{3}}{R_{1}} \frac{1}{1+s R_{3} C_{2}}
$$

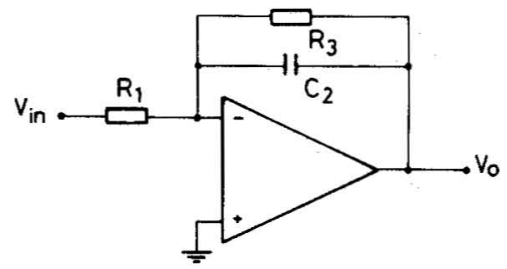

Fig. 2A Continuous-time damped integrator

Again, positive or negative integrators can be obtained in the differential form, as shown in Fig. 2B, by simply controlling the proper connection of the input signal pair.

Following the same approach as for the lossless bilinear 
integrator of Fig. 1C, the bilinear damped integrator (positive or negative) is obtained from the circuit of Fig. 2C. The $z$-domain transfer function of this integrator is

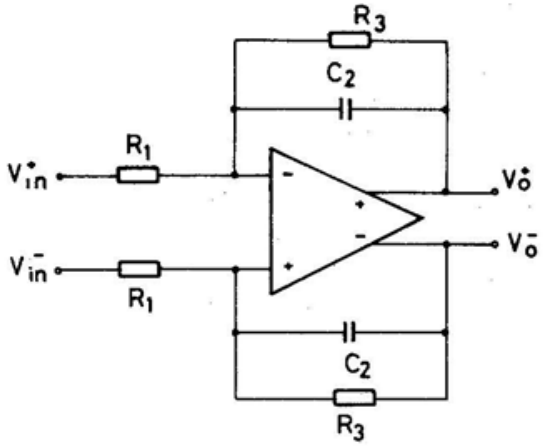

Fig. 2B Differential damped integrator

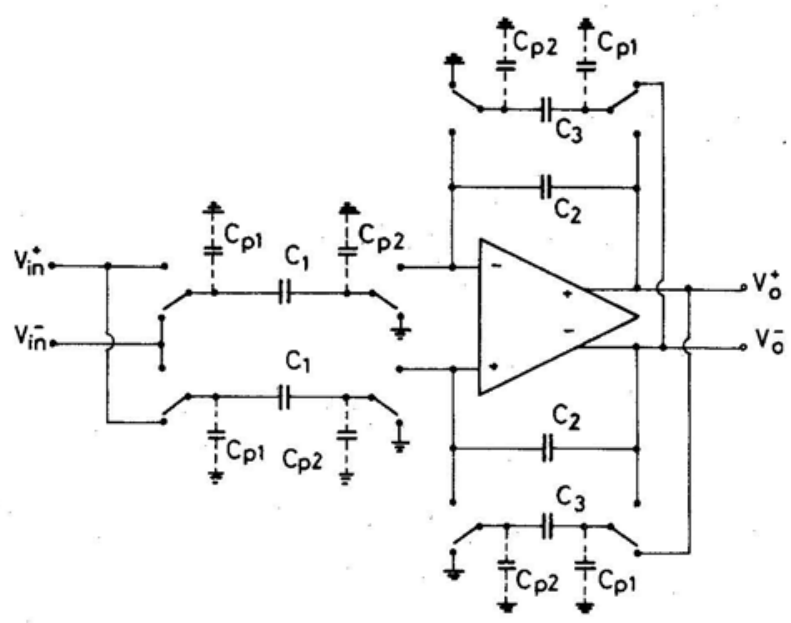

Fig. 2C Bilinear SC damped integrator

given by

$$
H_{2}(z)=-\frac{C_{1}}{C_{3}} \frac{1}{1+\frac{C_{2}}{C_{3}} \frac{z-1}{z+1}}
$$

which can be regarded as derived from eqn. 8 using the bilinear $s$ to $z$ transform and by applying

$$
\begin{aligned}
& C_{1}=\frac{T}{2 R_{1}} \\
& C_{3}=\frac{T}{2 R_{3}}
\end{aligned}
$$

maintaining the integrating capacitor $C_{2}$ at the same value as in the continuous-time case.

The summing operation within an integrator can be performed by means of capacitors, as illustrated in Fig. 3.

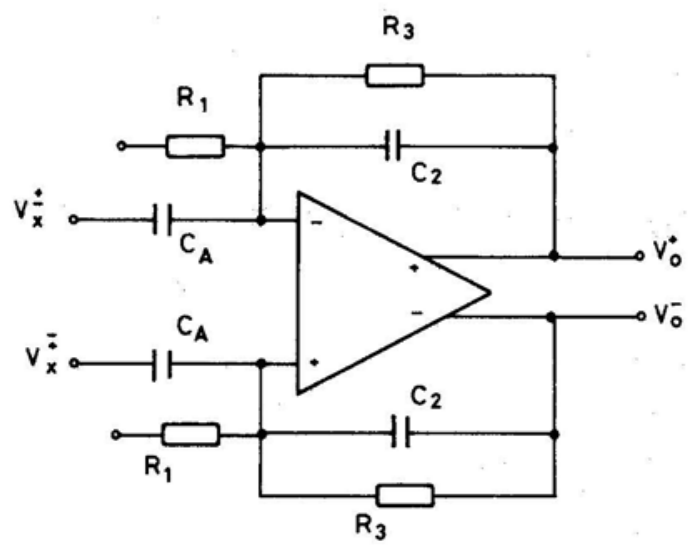

Fig. 3 Positive or negative summing stage
In SC implementations, the substitution of $R_{1}$ and eventually also of $R_{3}$ with the equivalent bilinear $\mathrm{SC}$ structures, does not cause any modification in the transfer function from $V_{x}$ to the output, as in any case

$$
K \equiv \frac{V_{o}^{+}-V_{o}^{-}}{V_{x}^{ \pm}-V_{x}^{\mp}}=\mp \frac{C_{A}}{C_{2}}
$$

So, either positive or negative amplification gain constants $K$ are obtained, where the gain factor $|K|$ is set by the capacitive ratio $C_{A} / C_{2}$.

All the SC integrators described above can be operated at a sampling frequency equal to twice the clock frequency $f_{c}$ if we duplicate the SC structures and connect them as shown in Fig. 4. The two structures connected at the same

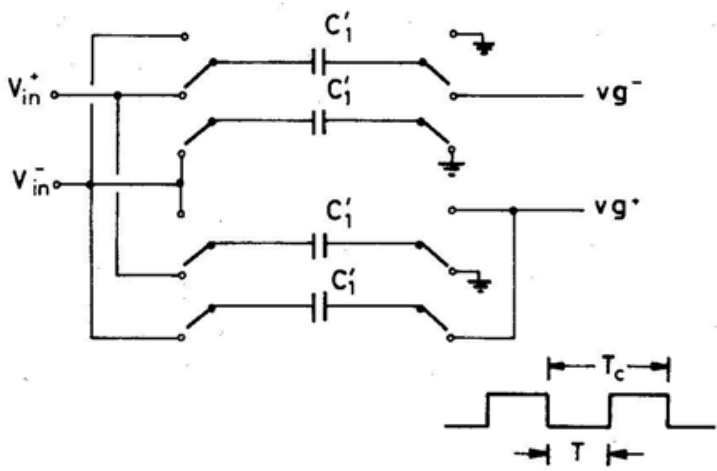

Fig. 4 Doubled-structure SC bilinear resistor

input of the operational amplifier work in turn in charging and discharging phases during the same half clock period $T_{c}=1 / f_{c}$, thus doubling the actual sampling frequency $f_{s}=1 / T$. In this case, $T$ being the holding signal period, the correspondence between the doubled SC structure and the equivalent analogue resistor $R_{1}$ is given by

$$
C_{1}^{\prime}=\frac{T}{2 R_{1}}=\frac{T_{c}}{4 R_{1}}
$$

Similarly, damped integrators can be implemented.

\section{Design of bilinear SC ladder filters}

As is well known, the bilinear $s$ to $z$ transformation given by eqn. 2 implies a warping effect between the continuoustime angular frequency $\Omega$ and the discrete-time angular frequency $\omega$, given by

$$
\Omega=\frac{2}{T} \tan \frac{\omega T}{2}
$$

and no distortion error is associated with this transformation. This means that an equiripple analogue design retains its equipripple response, while the whole axis $j \Omega$ from $-\infty$ to $+\infty$ is mapped into the unit circle in the $z$-plane, according to the relationship $z=e^{j \omega T}$.

It is worthwhile noting that a zero is naturally introduced at the Nyquist frequency $1 / 2 T$ for $z=-1$; this is an advantage of high-selectivity filter design over LDIbased design, where only the interval $|f|<1 / \pi T$ is mapped onto the unit circle.

The warping effect can be disregarded in the range of $\omega T \ll 1$, as in this case $\Omega \approx \omega$, and a continuous-time filter in the $s$-domain can be designed with exactly the same final specifications as required by the assigned mask.

Otherwise, if $\omega T$ is not much smaller than unity, the warping effect has to be taken into account and the desired final mask has to be prewarped according to eqn. 13. If $\omega_{i}$ are the characteristic frequencies of the filter to be 
designed, the analogue prototype must be chosen so as to meet a mask, where the characteristic frequencies $\Omega_{i}$ are obtained as

$$
\Omega_{i}=\frac{2}{T} \tan \frac{\omega_{i} T}{2}
$$

Furthermore, when $\omega T$ is not much smaller than unity, another effect is introduced by the SC filter with respect to the analogue prototype, that is the $\sin (\omega T / 2) /(\omega T / 2)$ distortion, caused by the inherent holding at the output stage of any SC network. To take this effect into account, the analogue prototype must be designed to have a frequency response $H(j \Omega)$ tilted-up by a factor $x / \sin x$ where $x=\tan ^{-1}(\Omega T / 2)$. This can be done either by considering the effect by means of proper direct approximation methods [22], or by optimising the final $R L C$ network obtained without the $x / \sin x$ distortion [23]. We have chosen the second approach in the example given in the following Section.

When the final reference $R L C$ prototype has been obtained, the standard flow-graph rules are followed [1] to derive the ladder chain of integrators. Each integrator is then implemented with the differential blocks considered in Section 2, with the integrating time constants $\tau_{i}$ set as

$$
\tau_{i}=T \frac{C_{I}}{2 C_{\xi}}
$$

where $C_{I}$ is the integrating capacitor and $C_{\xi}$ is the capacitor value of the SC structure.

From a different point of view, this corresponds to an element-to-element substitution of the resistive components $R_{i}$ of the active continuous-time ladder, where the bilinear differential SC structures (with $C_{\xi}=T / 2 R_{i}$ ) are properly connected for obtaining positive or negative bilinear SC resistors.

\section{Example}

The approach described above is shown in this Section in an example. The specifications of the filter to be designed are as follows:

Passband: $F_{l}=90 \mathrm{kHz} ; F_{h}=110 \mathrm{kHz}$

Ripple: $0.2 \mathrm{~dB}$ max.

Stopband: $F_{s l}=70 \mathrm{kHz} ; F_{s h}=130 \mathrm{kHz}$; Attenuation: $35 \mathrm{~dB}$

Sampling frequency $1 / T=500 \mathrm{kHz}$

First, all the corner frequencies of the specified mask have to be prewarped according to eqn. 14 , giving

$$
\begin{aligned}
& F_{l}^{\prime}=101.0 \mathrm{kHz} \\
& F_{h}^{\prime}=131.6 \mathrm{kHz} \\
& F_{s l}^{\prime}=74.9 \mathrm{kHz} \\
& F_{s h}^{\prime}=169.5 \mathrm{kHz}
\end{aligned}
$$

As a second step, we must choose the analogue $R L C$ prototype. Using the classical lowpass to bandpass transformation, the normalised lowpass $L C$ ladder prototype $C$ 04-01-20 has been chosen. Such a filter has a passband ripple much lower than the required one; this is to prevent eventual imperfections in the optimisation step. The circuit with denormalised values is given in Fig. 5.

By transforming the lowpass network into the bandpass one, we obtain the doubly terminated $L C$ topology shown in Fig. 6. This filter will show a flat passband between $F_{l}^{\prime}$ and $F_{h}^{\prime}$. Then, as a third step, the values of the components must be modified so that the filter can satisfy the same passband mask, but tilted up by the factor $x / \sin x$ with $x=\tan ^{-1}(\Omega / 1 M H z)$. Such a modified passband mask is shown in Fig. 7, where also the discretisation in narrow

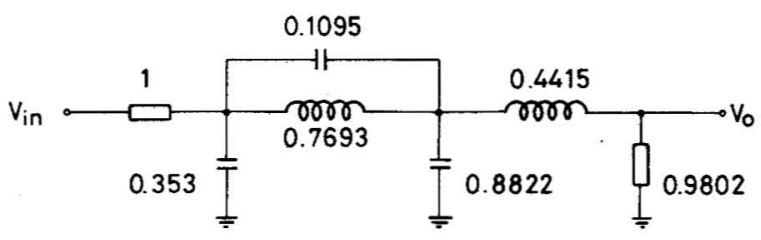

Fig. 5 Lowpass prototype

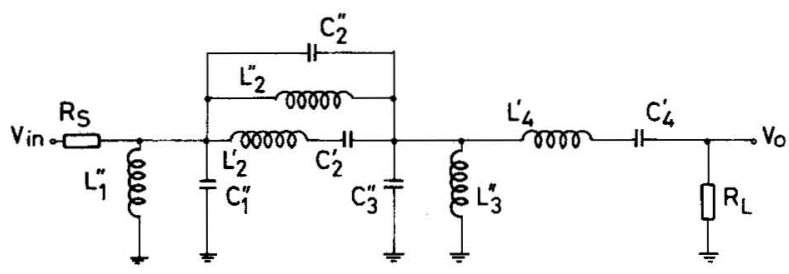

Fig. 6 Bandpass transformed prototype (denormalised values)

$$
\begin{array}{ll}
\boldsymbol{R}_{\mathrm{s}}=1 \Omega & \boldsymbol{C}_{2}^{\prime}=0.477 \mu \mathrm{F} \\
L_{1}^{\prime \prime}=1.04 \mu \mathrm{H} & L_{3}^{\prime \prime}=0.416 \mu \mathrm{H} \\
C_{1}^{\prime \prime}=1.832 \mu \mathrm{F} & C_{3}^{\prime \prime}=4.579 \mu \mathrm{F} \\
L_{2}^{\prime \prime}=3.352 \mu \mathrm{H} & L_{4}^{\prime}=2.292 \mu \mathrm{H} \\
C_{2}^{\prime \prime}=0.568 \mu \mathrm{F} & C_{4}^{\prime}=0.831 \mu \mathrm{F} \\
L_{2}^{\prime}=3.993 \mu \mathrm{H} & R_{L}=0.9802 \Omega
\end{array}
$$

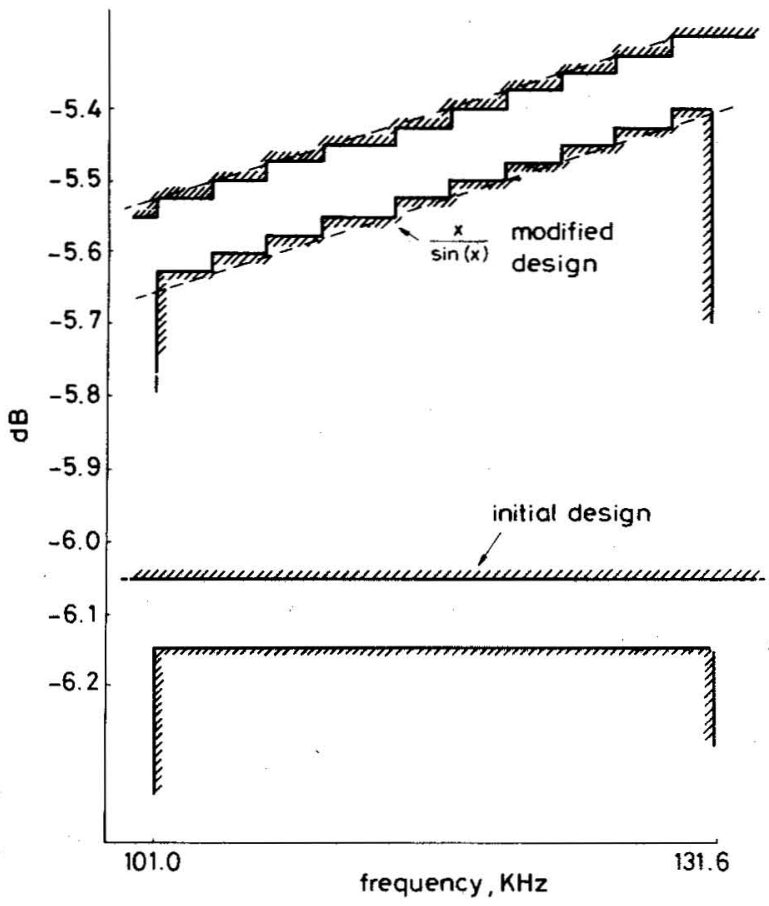

Fig. 7 Passhand mask corrected for the $x / \sin \times$ factor

flat subintervals is reported, as required for the computer optimising design [23].

After the optimisation [23] we obtain the values of the components shown in Fig. 8.

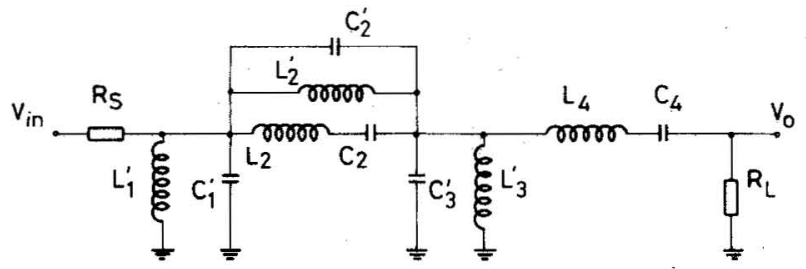

Fig. 8 Prototype with the final values after optimisation

$\begin{array}{ll}R_{s}=1 \Omega & C_{2}=0.485 \mu \mathrm{F} \\ L_{1}^{\prime}=1.011 \mu \mathrm{H} & L_{3}^{\prime}=0.393 \mu \mathrm{H} \\ C_{1}^{\prime}=1.808 \mu \mathrm{F} & C_{3}^{\prime}=4.579 \mu \mathrm{F} \\ L_{2}^{\prime}=3.363 \mu \mathrm{H} & L_{4}=2.291 \mu \mathrm{H} \\ C_{2}^{\prime}=0.568 \mu \mathrm{F} & C_{4}=0.831 \mu \mathrm{F} \\ L_{2}=3.982 \mu \mathrm{H} & R_{L}=1.165 \Omega\end{array}$


By using signal flow-graph techniques, we describe the electrical behaviour of the final bandpass topology. First, we open the inductor loop [24] as well as the capacitive loop, obtaining the circuit of Fig. 9. The flow-graph shown

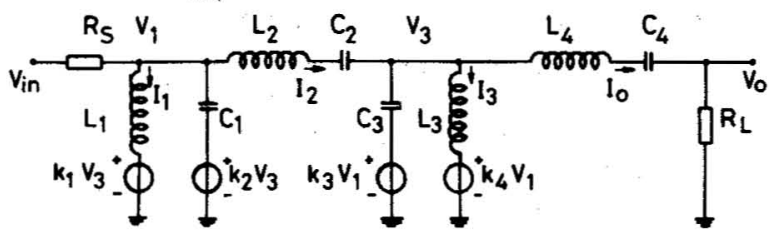

Fig. $9 \quad R L C$ prototype reduced with equivalent Thévenin networks

$k_{1}=\frac{L_{1}^{\prime}}{L_{1}^{\prime}+L_{2}^{\prime}}=0.231$

$k_{2}=\frac{C_{2}^{\prime}}{C_{1}^{\prime}+C_{2}^{\prime}}=0.239$

$k_{3}=\frac{C_{2}^{\prime}}{C_{2}^{\prime}+C_{3}^{\prime}}=0.11$

$k_{4}=\frac{L_{3}^{\prime}}{L_{2}^{\prime}+L_{3}^{\prime}}=0.105$

$$
\begin{array}{ll}
L_{1}=L_{1}^{\prime} / / L_{2}^{\prime} & =0.777 \mu \mathrm{H} \\
C_{1}=C_{1}^{\prime}+C_{2}^{\prime} & =2.376 \mu \mathrm{F} \\
L_{2} & =3.982 \mu \mathrm{H} \\
C_{2} & =0.485 \mu \mathrm{F} \\
L_{3}=L_{2}^{\prime} / / L_{3}^{\prime} & =0.352 \mu \mathrm{H} \\
C_{3}=C_{2}^{\prime}+C_{3}^{\prime} & =5.147 \mu \mathrm{F} \\
L_{4} & =2.291 \mu \mathrm{H} \\
C_{4} & =0.831 \mu \mathrm{F} \\
R_{L} & =1.165 \\
R_{S} & =1
\end{array}
$$

in Fig. 10 corresponds to this modified circuit, where the two branches $k_{2}$ and $k_{3}$ realise the transmission zero pair.

Finally, by using the blocks considered in Section 2, we obtain the circuit of Fig. 11, where symbolic blocks have been used for the sake of simplicity. Inverting SC blocks are used for positive branches and vice versa for negative ones to take into account the sign inversion of the active integrators.

Each time constant is then set according to eqn. 15 or, setting all the switched capacitors of unit value, the integrating capacitors are set according to eqn. 15 . The capacitor values have been scaled for dynamic range considerations, following the classical method [19]; the passband gain of the filter is consequently set at zero dB.

The capacitor values are summarised in Table 1: the first column reports the starting values, the second column reports the values after the dynamic range optimisation, and the third column reports the values normalised to unity capacitance [19]. The total capacitance is 208.5 with a capacitor ratio spread of $1: 13.3$.

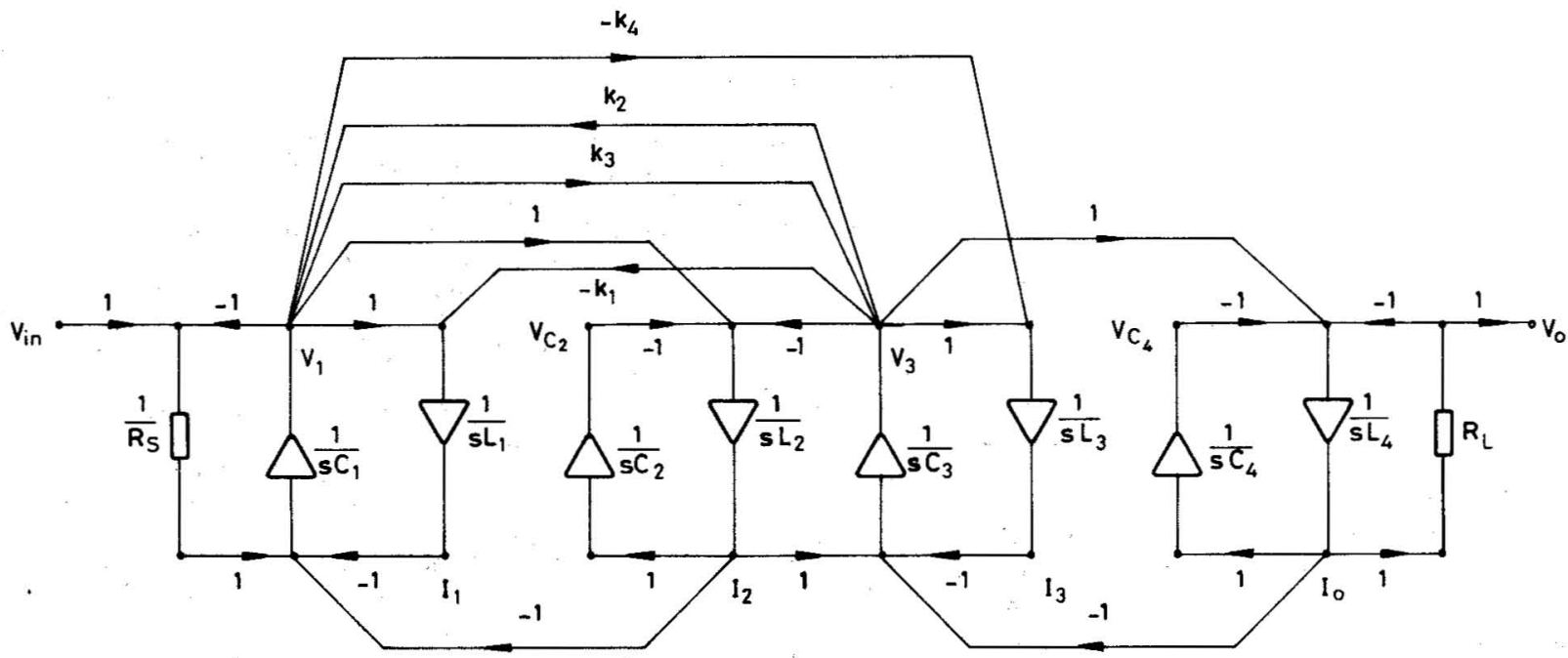

Fig. 10 Flow-graph corresponding to the circuit of Fig. 9

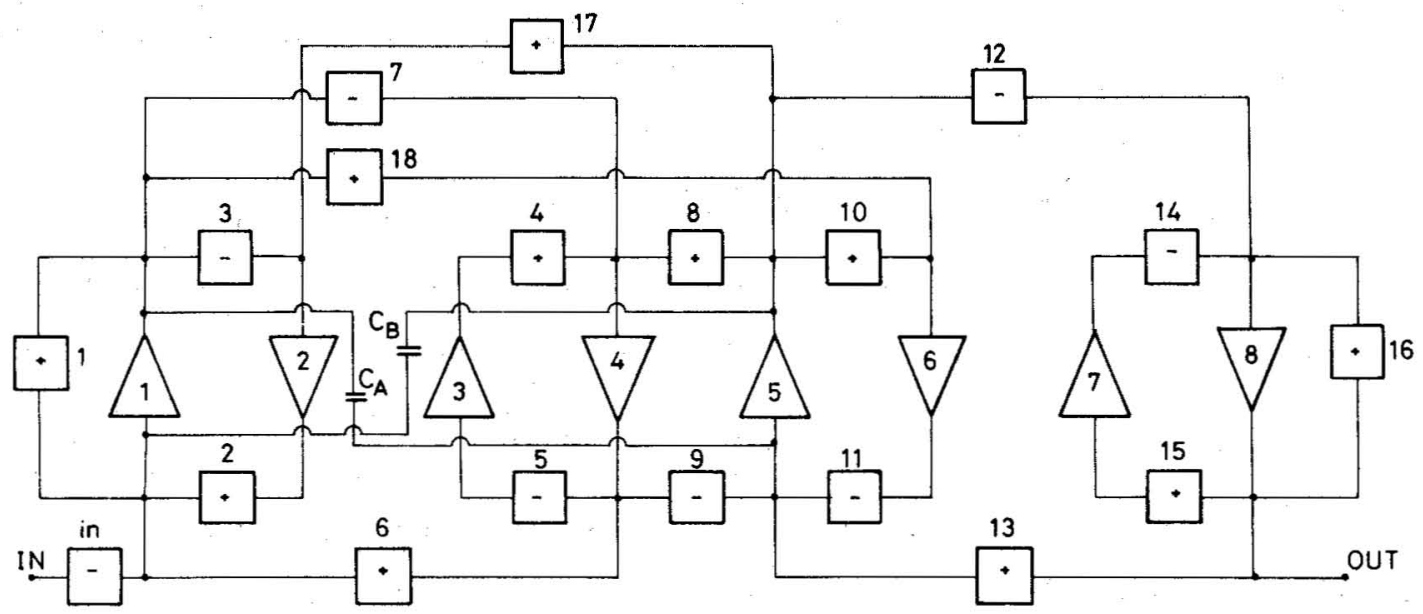

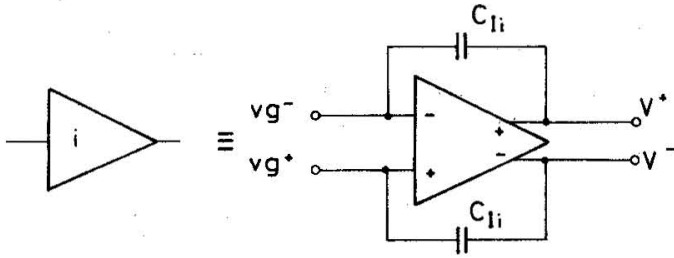

Fig. 11 Final SC bandpass filter (schematical representation)
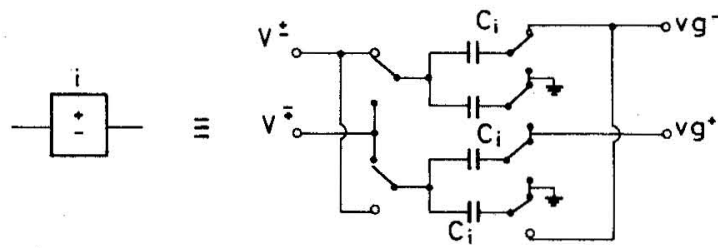
Table 1: Capacitor values

\begin{tabular}{llll}
\hline \multicolumn{3}{c}{ Switched-capacitors } \\
\hline$C_{\text {in }}$ & 1 & 1 & 2 \\
$C_{1}$ & 1 & 0.8785 & 1.75 \\
$C_{2}$ & 1 & 2.1815 & 4.37 \\
$C_{3}$ & 1 & 0.8785 & 6.89 \\
$C_{4}$ & 1 & 3.2916 & 5.96 \\
$C_{5}$ & 1 & 1 & 1 \\
$C_{6}$ & 1 & 1 & 2 \\
$C_{7}$ & 1 & 0.8785 & 1.59 \\
$C_{8}$ & 1 & 0.5515 & 1 \\
$C_{9}$ & 1 & 1 & 3.19 \\
$C_{10}$ & 1 & .05515 & 6 \\
$C_{11}$ & 1 & 2.7510 & 8.77 \\
$C_{12}$ & 1 & 0.5515 & 1 \\
$C_{13}$ & 1 & 0.4274 & 1.36 \\
$C_{14}$ & 1 & 0.8929 & 1.79 \\
$C_{15}$ & 1 & 0.4274 & 1 \\
$C_{16}$ & 1.1650 & 0.4979 & 1 \\
$C_{17}$ & 0.2311 & 0.1275 & 1 \\
$C_{18}$ & 0.1046 & 0.0919 & 1 \\
\hline
\end{tabular}

Integrating capacitors

\begin{tabular}{lllc}
\hline$C_{I 1}$ & 2.3764 & 2.0877 & 4.18 \\
$C_{/ 2}$ & 0.7773 & 1.6957 & 13.3 \\
$C_{/ 3}$ & 0.4850 & 1.5964 & 1.59 \\
$C_{14}$ & 3.9820 & 3.9820 & 7.22 \\
$C_{/ 5}$ & 5.1474 & 2.8387 & 9.05 \\
$C_{16}$ & 0.3519 & 0.9680 & 10.53 \\
$C_{17}$ & 0.8313 & 0.7422 & 1.73 \\
$C_{18}$ & 2.2910 & 0.9791 & 1.96 \\
\hline
\end{tabular}

\begin{tabular}{llll} 
& \multicolumn{3}{c}{ Transmission zeros } \\
\hline$C_{A}$ & 0.5684 & 0.3135 & 1 \\
$C_{B}$ & 0.5684 & 0.4993 & 1 \\
\hline
\end{tabular}

For practical implementation, the circuit of Fig. 11 can be optimised in terms of the switch count. Combining the actions of the switches incident at the same operational amplifier, there are eight switches per operational amplifier; moreover, two additional toggles are required in the input structure.
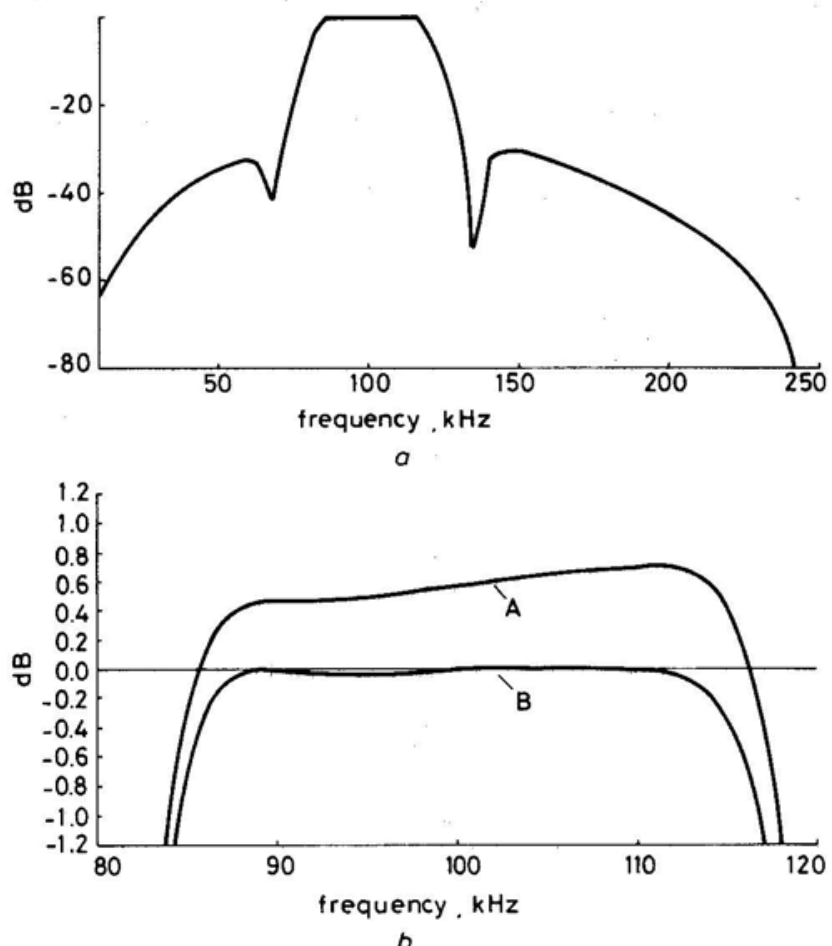

Fig. 12 Passband response of the SC filter

$a$ Whole response

$b$ Details in the passband excluding (curve A), and including (curve B) the $\sin x / x$ effect
The transmission zeros are realised by the capacitors $C_{A}$ and $C_{B}$ shown in the schematic of Fig. 11; their values are set by the amplification constants $k_{2}$ and $k_{3}$ given from the flow-graph of Fig. 10, according to eqn. 11.

The frequency response of the filter has been calculated using the SWITCAP [26] package and it is shown in Fig. $12 a$, and the details of the passband are shown in Fig. $12 b$. Here, the actual sampled-data transfer function of the circuit evaluated into the unit circle in $z$-domain is represented by curve $A$. When the holding effect is also taken into account (curve B), the frequency response of the designed filter in the passband is sufficiently flat, and satisfies the required design mask.

\section{$5 \quad$ Acknowledgment}

Work partially supported by the Italian National Council of Research, CNR.

\section{$6 \quad$ References}

1 JACOBS, G.M., ALLSTOT, D.J., BRODERSEN, R.W., and GRAY, P.R.: 'Design techniques for MOS switched capacitor ladder filters', IEEE Trans., 1978, CAS-25, (12), pp. 1014-1020

2 MARTIN, K., and SEDRA, A.S.: 'Designing leap-frog and SFG filters with optimum dynamic range', Proc. IEEE, 65, 1977, pp. 1210-1211

3 ORCHARD, H.J.: 'Inductorless filters', Electron. Lett, 1966, 2, pp. 224-225

4 GHAUSI, M.S., and LAKER, K.R., (Eds). 'Modern filter design' (Prentice Hall, NJ, USA, 1981)

5 BRUTON, L.T.: 'Low-sensitivity digital ladder filters' IEEE Trans., 1975, CAS-22, (3), pp. 168-176

6 GREGORIAN, R., and NICHOLSON, W.E., jr: 'CMOS switchedcapacitor filters for a PCM voice CODEC', IEEE J. Solid-State Circuits, 1979, SC-14, (6), pp. 970-980

7 CHOI, T.C., and BRODERSEN, R.W.: 'Considerations for HighFrequency Switched-Capacitor Ladder Filters', IEEE Trans., 1980, CAS-27, (6), pp. 545-552

8 DAVIS, R.D., and TRICK, T.N.: 'Optimum design of lowpass switched-capacitor ladder filters', ibid., 1980, CAS-27, (6), pp. 522-527

9 SCANLAN, S.O.: 'Analysis and synthesis of switched-capacitor statevariable filters', ibid., 1981, CAS-28, (2), pp. 85-93

10 BAHER, H., and SCANLAN, S.O.: 'Exact synthesis of bandpass switched-capacitor LDI ladder filters', ibid., 1984, CAS-31, (4), pp. $342-348$

11 DATAR, R.B., and SEDRA, A.S.: 'Exact design of strays-insensitive switched-capacitor ladder filters', ihid., 1983, CAS-30, (12), pp. 888-897

12 LIU, E.S.K., TURNER, L.E., and BRUTON, L.T.: 'Exact synthesis of LDI and LDD ladder filters', ibid., 1984, CAS-31, (4), pp. 369-381

13 LEE, M.S., and CHANG, C.: 'Switched-capacitor filters using the LDI and the bilinear transformations', ibid., 1981, CAS-28, (4), pp. 265-270

14 TAYLOR, J.T., and MAVOR, J.: 'Exact design of stray-insensitive switched-capacitor lowpass ladder filters from unit-element prototypes', Electron. Lett., 1984, 20, pp. 665-667

15 TEMES, G.C., ORCHARD, H.J., and JAHANBEGLOO, M.: 'Switched-capacitor filter design using the bilinear z-transform', IEEE Trans., 1978, CAS-25, (12), pp. 1039-1044

16 VELAZQUEZ-RAMOS, J., STEPHENSON, F.W., RIAD, S.M., and RIAD, A.A.: 'High frequency switched-capacitor ladder filters'. 14th Southeastern Symp. on System Theory, 1982, Dig. of Techn. Papers, pp. 114-117

17 ERIKSSON, S., and AKHLAGHI, H.: 'Noninverting parasiticcompensated bilinear SC integrator with only one amplifier', Electron. Lett., 1983, 19, pp. 450-452

18 MONTECCHI, F., and MALOBERTI, F.: 'Switched-capacitor ladder filters for high frequency applications via bilinear resistor modeling'. 26th Midwest Symp. on Circuits and Systems, 1983, Dig. of Techn. Papers, pp. 445-447

19 LEE, M.S., TEMES, G.C., CHANG, C., and GHADERI, M.B.: 'Bilinear switched-capacitor ladder filters', IEEE Trans., 1981, CAS-28, (8), pp. $811-821$

20 MARTIN, K.: 'Improved circuits for the realization of switchedcapacitor filters', ibid., 1980, CAS-27, (4), pp. 237-244 
21 SENDEROWICZ, D., DREYER, S.F., HUGGINS, J.M., RAHIM, C.F., and LABER, C.A.: 'A family of differential NMOS analog circuits for a PCM codec filter chip', IEEE J. Solid-State Circuits, 1982, SC-17, pp. 1014-1023

22 NAKHLA, M.S.: 'Approximation of lowpass filters with frequencydependent input gain characteristic', IEEE Trans., 1979, CAS-26, (3), pp. 198-202

23 HALASZ, E.: 'Design centering and tolerancing, considering environmental effects via a new type minimax optimisation', IEE Proc. G, Electron Circuits \& Syst., 1982, 129, pp. 134-138

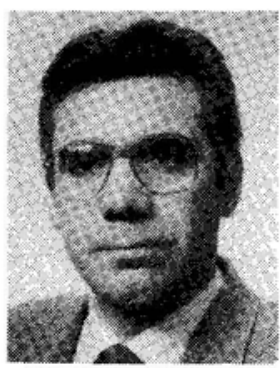

Franco Maloberti was born in Parma in 1945. He received the Laurea degree in Physics in 1968. Since 1969 he has been with the Department of Electronics of the University of Pavia, where he is now an Associate Professor. His research interests include the design of analogue MOS integrated circuits and switched-capacitor circuits.

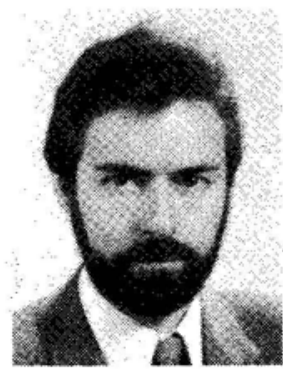

Federico Montecchi was born in Terni, Italy, in 1950 . He received the Laurea degree in electronics engineering from the University of Pavia in 1973. Since 1975 he has been with the Department of Electronics of the Faculty of Engineering, University of Pavia, where he is now an Associate Professor. His scientific interests were previously concerned with the noise characterisation of semiconductor components and circuits, mainly CCD, while he is now working in the area of switched-capacitor networks.
24 GREGORIAN, R., and FANG, S.C.: 'Ladder simulation SC filters with inductor loops', Electron. Lett., 1981, 17, pp. 786-788

25 BANU, M., and TSIVIDIS, Y.: 'Fully integrated active RC filters in MOS technology', IEEE J. Solid-State Circuits, 1983, SC-18, pp. 644-651

26 FANG, S.C., TSIVIDIS, Y.P., and WING, O.: 'SWITCAP: a switched-capacitor network analysis program-Part I and II', IEEE Circuits \& Syst. Mag., Sep. 1983, pp. 4-10, and ibid., Dec. 1983, pp. $41-46$

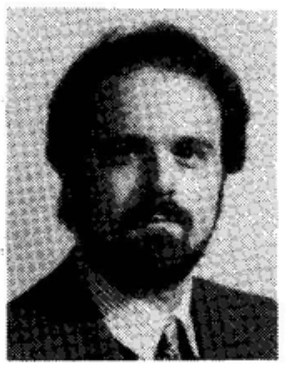

Guido Torelli was born in Rome, Italy, in 1949. He graduated in Electronic Engineering in 1973 from the University of Pavia, where after graduating he worked for one year as a researcher in the Institute of Electronics. In 1974 he joined SGS, where he was engaged as a design engineer in MOS ICs Development Department. He is now a Project Leader in the field of MOS ICs for Consumer Market. His design interest ranges both in digital and in analog circuits.

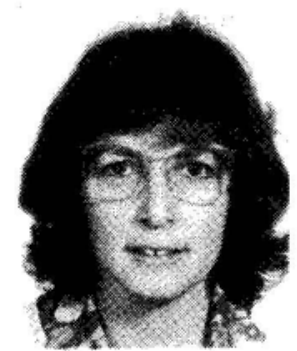

Edith Halász was born in Budapest, Hungary. She received a Diploma in electrical engineering from the Technical University of Budapest. Her research interests are in the areas of network theory, optimisation methods and computer-aided design. She has been a consultant to various electronic industries in the areas of CAD of LC filters, SC filters, equalizers and tolerance centering of LC filters. She received the degree of Doctor Techn. from the Technical University of Budapest in 1972, and the degree of Candidate of Sciences in circuit theory from the Hungarian Academy of Sciences, Budapest in 1983. Dr Halász works for the Technical University of Budapest in the Institute of Telecommunication and Electronics. 